\title{
Nash equilibrium as an expression of self-referential reasoning
}

Citation for published version (APA):

Perea ý Monsuwé, A. (2006). Nash equilibrium as an expression of self-referential reasoning. METEOR, Maastricht University School of Business and Economics. METEOR Research Memorandum No. 035 https://doi.org/10.26481/umamet.2006035

Document status and date:

Published: 01/01/2006

DOI:

10.26481/umamet.2006035

Document Version:

Publisher's PDF, also known as Version of record

\section{Please check the document version of this publication:}

- A submitted manuscript is the version of the article upon submission and before peer-review. There can be important differences between the submitted version and the official published version of record.

People interested in the research are advised to contact the author for the final version of the publication, or visit the DOI to the publisher's website.

- The final author version and the galley proof are versions of the publication after peer review.

- The final published version features the final layout of the paper including the volume, issue and page numbers.

Link to publication

\footnotetext{
General rights rights.

- You may freely distribute the URL identifying the publication in the public portal. please follow below link for the End User Agreement:

www.umlib.nl/taverne-license

Take down policy

If you believe that this document breaches copyright please contact us at:

repository@maastrichtuniversity.nl

providing details and we will investigate your claim.
}

Copyright and moral rights for the publications made accessible in the public portal are retained by the authors and/or other copyright owners and it is a condition of accessing publications that users recognise and abide by the legal requirements associated with these

- Users may download and print one copy of any publication from the public portal for the purpose of private study or research.

- You may not further distribute the material or use it for any profit-making activity or commercial gain

If the publication is distributed under the terms of Article $25 \mathrm{fa}$ of the Dutch Copyright Act, indicated by the "Taverne" license above, 
Andrés Perea

Nash Equilibrium as an Expression of SelfReferential Reasoning

$\mathrm{RM} / 06 / 035$

JEL code: C72

METE@R

Maastricht research school of Economics of TEchnology and ORganizations

Universiteit Maastricht

Faculty of Economics and Business Administration P.O. Box 616

NL - 6200 MD Maastricht

phone : ++31433883830

fax : $\quad++31433884873$ 



\title{
Nash Equilibrium as an Expression of Self-Referential Reasoning
}

\author{
Andrés Perea \\ Maastricht University
}

This Version: September 2006

\begin{abstract}
Within a formal epistemic model for simultaneous-move games, we present the following conditions: (1) belief in the opponents' rationality (BOR), stating that a player should believe that every opponent chooses an optimal strategy, (2) self-referential beliefs (SRB), stating that a player believes that his opponents hold correct beliefs about his own beliefs, (3) projective beliefs (PB), stating that $i$ believes that $j$ 's belief about $k$ 's choice is the same as $i$ 's belief about $k$ 's choice, and (4) conditionally independent beliefs (CIB), stating that a player believes that opponents' types choose their strategies independently. We show that, if a player satisfies BOR, SRB and CIB, and believes that every opponent satisfies BOR, SRB, PB and CIB, then he will choose a Nash equilibrium strategy (that is, a strategy that is optimal in some Nash equilibrium). We thus provide a set of sufficient conditions for Nash equilibrium strategy choice. We also show that none of these seven conditions can be dropped.

Keywords: Nash equilibrium, epistemic game theory.

JEL Classification: C72
\end{abstract}

\author{
Andrés Perea \\ Maastricht University, Department of Quantitative Economics, \\ P.O. Box 616, 6200 MD Maastricht, The Netherlands. \\ E-mail: a.perea@ke.unimaas.nl \\ Web: http://www.personeel.unimaas.nl/a.perea/
}




\section{Introduction}

Since its introduction by John Nash (1951), the concept of Nash equilibrium has played an essential role in game theory and its various applications. It is therefore natural to look for reasonable conditions under which players may be expected to choose according to Nash equilibrium. Such conditions have been provided in many different settings. In learning theory one assumes that players play the game repeatedly, and one can find reasonable classes of learning rules that eventually lead players to choose according to some Nash equilibrium. Similarly, evolutionary game theory studies classes of replicator dynamics in the repeated game that converge to (special types of) Nash equilibria in the long run. If the game is to be played only once, one could think of a situation in which a mediator publicly announces the mixed strategy profile to the players, and recommends each player to play his strategy in this profile. Then, players may only be expected to follow their recommendation if the mixed strategy profile is a Nash equilibrium. One can also formulate sufficient conditions for Nash equilibrium in a static setting without mediators. See, for instance, Brandenburger and Dekel (1987), Aumann and Brandenburger (1995) and Asheim (2006). A key condition in each of these papers is that a player knows, or has a correct belief about, his opponents' beliefs about the other players' strategy choices.

A common feature of each of the models above is that it requires, either explicitly or implicitly, some sort of communication between players. In the learning models and evolutionary models players communicate by the actions they choose in the repeated game. It is this type of communication that allows them to converge to a Nash equilibrium in the long run. In the static models where players are assumed to have correct beliefs about the other players' beliefs, there seems to be a need for ex-ante communication between players in which they report their beliefs to others. Otherwise, there is no reason to expect that players should be right about the opponents' beliefs, even if common belief in rationality is imposed.

In this paper, we investigate whether we can find reasonable sufficient conditions for Nash equilibrium in static settings where there is no communication between players. In such a setting, a player can base his strategy choice solely upon his own beliefs about the opponents' choices and his own beliefs about the opponents' beliefs, since before making his decision he receives no information about what opponents do or believe. It thus makes sense to analyze the game from a single player's perspective, and see whether we can impose sensible conditions on this player's beliefs that lead to Nash equilibrium behavior.

As an illustration of our objective, consider the game in Figure 1, which is taken from Myerson (1991, page 94). Suppose that the game is played only once, and that player 1 (the row player) and player 2 (the column player) cannot communicate to each other. We will analyze this game completely from player 1's perspective. It can be shown that common belief in rationality does not exclude any of player 1's strategies, but that Nash equilibrium singles out strategy $b$. Consider, namely, a scenario in which

1. player 1 believes that player 2 chooses $d$, 


\begin{tabular}{|l|l|l|l|}
\hline & $d$ & $e$ & $f$ \\
\hline$a$ & 3,0 & 0,2 & 0,3 \\
\hline$b$ & 2,0 & 1,1 & 2,0 \\
\hline$c$ & 0,3 & 0,2 & 3,0 \\
\hline
\end{tabular}

Figure 1: Rationalizability versus Nash equilibrium

2. player 1 believes that player 2 believes that player 1 chooses $c$,

3. player 1 believes that player 2 believes that player 1 believes that player 2 chooses $f$,

4. player 1 believes that player 2 believes that player 1 believes that player 2 believes that player 1 chooses $a$,

5. player 1 believes that player 2 believes that player 1 believes that player 2 believes that player 1 believes that player 2 chooses $d$,

and so on. Then, player 1 respects common belief in rationality, since he believes that player 2 chooses rationally, believes that player 2 believes that player 1 chooses rationally, and so on. Since in this scenario it is rational for player 1 to choose $a$, we must allow for the strategy $a$ if we only impose common belief in rationality. Since strategies $a$ and $c$ play similar roles in this game, one can construct a similar scenario that leads to strategy choice $c$. Finally, strategy $b$ can be justified by a much simpler scenario in which

1. player 1 believes that player 2 chooses $e$,

2. player 1 believes that player 2 believes that player 1 chooses $b$,

3. player 1 believes that player 2 believes that player 1 believes that player 2 chooses $e$,

and so on. Summarizing, every strategy for player 1 can be justified by a belief hierarchy for player 1 that respects common belief in rationality. More generally, Tan and Werlang (1988) have shown that the strategies which can be chosen rationally in two-player games when imposing only common belief in rationality are exactly the rationalizable strategies in the sense of Bernheim (1984) and Pearce (1984). ${ }^{1}$

On the other hand, it can be shown that Nash equilibrium uniquely selects the strategy $b$ for player 1. In order to see this, it is helpful to consider Figure 2 which depicts the players' best reponse correspondences in this game. The first triangle should be read as follows. Every point in the triangle represents a probabilistic belief of player 1 about player 2's strategies. The

\footnotetext{
${ }^{1}$ For more than two players, one needs to impose that beliefs about different opponents be stochastically independent in order to obtain the equivalence with rationalizability.
} 

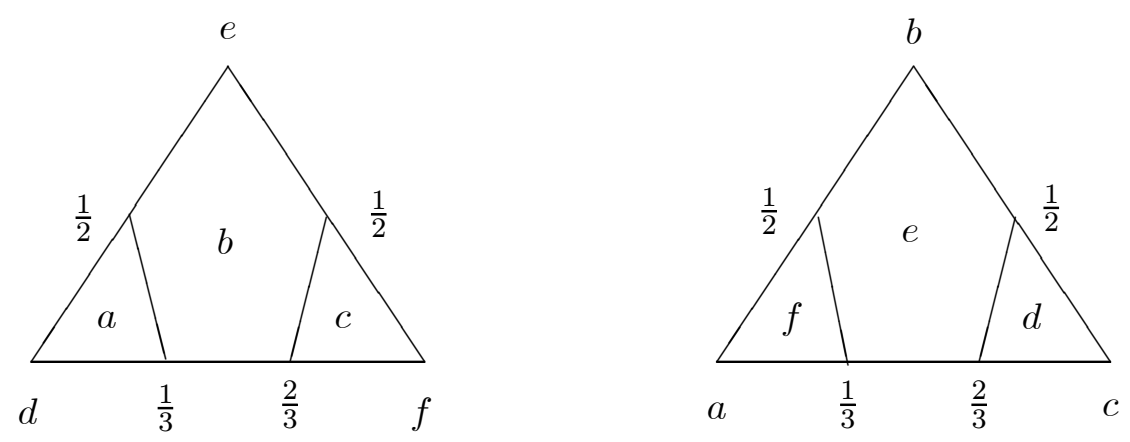

Figure 2: Best reponse correspondences for the game in Figure 1

three areas in this triangle represent the sets of beliefs for which the strategies $a, b$ and $c$ are optimal, respectively. Similarly for the second triangle. Consider a Nash equilibrium $\left(\mu_{1}, \mu_{2}\right)$ of the game, where $\mu_{i}$ is a probability distribution over $i$ 's strategies for $i=1,2$. We show that $\mu_{1}$ must assign probability 1 to $b$, and $\mu_{2}$ must assign probability 1 to $e$. Suppose, contrary to what we want to show, that $\mu_{1}$ assigns positive probability to $a$. Then, since $\left(\mu_{1}, \mu_{2}\right)$ is a Nash equilibrium, $a$ must be optimal for player 1 against $\mu_{2}$. By Figure 2, $\mu_{2}$ must then assign positive probability to $d$, and hence $d$ must be optimal against $\mu_{1}$. By Figure 2, $\mu_{1}$ must then assign positive probability to $c$, and hence $c$ must be optimal against $\mu_{2}$. So, both $a$ and $c$ should be optimal against $\mu_{2}$. However, from the first triangle it is clear that $a$ and $c$ cannot both be optimal against $\mu_{2}$, and hence we have a contradiction. By symmetry, one can similarly prove that $\mu_{1}$ cannot assign positive probability to $c$, and that $\mu_{2}$ cannot assign positive probability to $d$ or $f$. Hence, the only strategy that can rationally be chosen by player 1 in a Nash equilibrium is $b$.

Now, let us compare the scenario above which led to the non-Nash equilibrium choice $a$ with the simpler scenario that led to the Nash equilibrium choice $b$. One fundamental difference between both scenarios is that the first assumes that player 1 believes that player 2 is wrong about 1's belief, whereas the second scenario assumes that player 1 believes that player 2 is right about 1's belief. Namely, in the first scenario player 1 believes that player 2 chooses $d$, but he believes that player 2 believes that he believes that player 2 chooses $f$, and not $d$.

Even stronger, the choice $a$ can only be justified by scenarios in which player 1 believes that player 2 is wrong about 1's belief. In order to see this, assume that strategy $a$ would be optimal for player 1 . Then, player 1 must believe with positive probability that player 2 chooses $d$. In turn, player 2 can only rationally choose $d$ if he believes with positive probability that player 1 chooses $c$. Hence, player 1 must believe with positive probability that player 2 believes with positive probability that player 1 chooses $c$. However, there is no belief for player 1 for which both $a$ and $c$ are rational, and hence player 1 must believe with positive probability that player 
2 believes with positive probability that player 1's belief is different from his true belief.

Say that a belief hierarchy $\beta_{i}$ for player $i$ has self-referential beliefs if $\beta_{i}$ believes that every opponent $j$ believes that $i$ 's belief hierarchy is $\beta_{i}$. For the example in Figure 1, we may thus conclude that the difference between Nash equilibrium choices and rationalizable non-Nash equilibrium choices is that the former can be justified by a self-referential belief hierarchy, whereas the latter cannot. The question is whether this is true generally. In Theorem 4.5 we show that this is true for the class of two-player games. More precisely, we show in Theorem 4.5 that in every two-player game, a player who (1) believes in the opponent's rationality (BOR), (2) has self-referential beliefs (SRB), (3) believes that his opponent BOR, and (4) believes that his opponent has SRB, must choose a Nash equilibrium strategy. Here, by a Nash equilibrium strategy we mean a strategy that is optimal in some Nash equilibrium. Be careful: every strategy that is assigned positive probability in some Nash equilibrium is a Nash equilibrium strategy, but not vice versa. There are Nash equilibrium strategies that are not assigned positive probability in any Nash equilibrium (see the example in Figure 3).

The four conditions above are no longer enough if we turn to more than two players. Similarly to other foundations for Nash equilibrium in the literature, we encounter the following two problems for more than two players: (a) We must guarantee that player $i$ 's belief about opponent $j$ 's strategy choice should be stochastically independent from $i$ 's belief about opponent $k$ 's strategy choice, and (b) we must guarantee that player $i$ believes that opponents $j$ and $k$ hold the same belief about player l's strategy choice. In this paper, we guarantee these events by imposing conditionally independent beliefs and projective beliefs. Conditionally independent beliefs (CIB) is taken from Brandenburger and Friedenberg (2006), and says that for any given profile of belief hierarchies for $i$ 's opponents, player $i$ 's belief about his opponents' strategy choices conditional on this profile of belief hierarchies should be uncorrelated. In other words, any correlation in $i$ 's belief about his opponents' strategy choices should be due to correlation in his belief about the opponents' belief hierarchies. The idea behind this condition is that players with fixed belief hierarchies are assumed to choose their strategies independently. Projective beliefs (PB), in turn, states that player $i$ believes that $j$ 's belief about player $k$ is the same as his own belief about player $k$. That is, player $i$ projects his own belief about player $k$ on player $j$. In Theorem 4.5 we show that for three players or more, a player who (1) BOR, (2) has SRB, (3) believes that his opponents BOR, (4) believes that his opponents have SRB, and, in addition, (5) has CIB, (6) believes that his opponents have PB, and (7) believes that his opponents have CIB, must choose a Nash equilibrium strategy. At this stage, one may wonder why we did not impose that the player himself has PB. The reason is that this property follows from the assumptions that the player has SRB and believes that every opponent has PB (see Lemma 4.2).

The outline of this paper is as follows: In Section 2 we present our epistemic model. Section 3 formally introduces the notions of BOR, SRB, PB and CIB. In Section 4 we show that every player who satisfies the conditions (1) - (7) above must choose a Nash equilibrium strategy. In Theorem 4.6 we prove the converse of this result, namely that every Nash equilibrium strategy can rationally be chosen by a player who satisfies these conditions (1) - (7). In Section 5 we 
prove that none of these seven conditions can be dropped in Theorem 4.5. In particular, this implies that the seven conditions are logically independent. In Section 6 we compare our result with other foundations of Nash equilibrium provided in Aumann and Brandenburger (1995), Asheim (2006) and Brandenburger and Dekel (1987).

\section{Epistemic Model}

Let $I$ be a finite set of players. A finite game is a tuple $\Gamma=\left(S_{i}, u_{i}\right)_{i \in I}$, where $S_{i}$ is the finite set of strategies for player $i$, and $u_{i}: \times_{j \in I} S_{j} \rightarrow \mathbb{R}$ is player $i$ 's utility function. We shall assume throughout that a player believes that the utility functions are as specified by $\Gamma$, that a player believes that all players believe this, and so on. For every finite set $X$, let $\Delta(X)$ denote the set of probability distributions on $X$.

Definition 2.1. (Epistemic model) A finite epistemic model for the game $\Gamma$ is a tuple

$$
\mathcal{M}=\left(T_{i}, \beta_{i}\right)_{i \in I}
$$

where, for every player $i, T_{i}$ is a finite set of types, and $\beta_{i}$ is a one-to-one function from $T_{i}$ to $\Delta\left(S_{-i} \times T_{-i}\right)$.

Here, $S_{-i}$ is a short way to write $\times_{j \neq i} S_{j}$, and similarly for $T_{-i}$. The interpretation of $\beta_{i}$ is that for every type $t_{i} \in T_{i}$, the image $\beta_{i}\left(t_{i}\right)$ denotes $t_{i}$ 's probabilistic belief about the opponents' strategy choices and types. For every event $E \subseteq S_{-i} \times T_{-i}$ for player $i$ and every number $p \in[0,1]$, we say that type $t_{i}$ believes the event $E$ with probability $p$ if $\beta_{i}\left(t_{i}\right)(E)=p$. We say that $t_{i}$ believes $E$ if $\beta_{i}\left(t_{i}\right)(E)=1$. For instance, we say that $t_{i}$ believes that player $j$ has type $t_{j}$ if $t_{i}$ assigns probability 1 to the set of strategy-type profiles in $S_{-i} \times T_{-i}$ where player $j$ 's type is $t_{j}$.

\section{Restrictions on Beliefs}

In this section we discuss four conditions that one may impose on a player's beliefs: belief in the opponents' rationality, self-referential beliefs, projective beliefs and conditionally independent beliefs. We first need some additional terminology. For every type $t_{i}$ and strategy profile $s_{-i} \in S_{-i}$, we denote by $\beta_{i}\left(t_{i}\right)\left(s_{-i}\right)$ the probability that the belief $\beta_{i}\left(t_{i}\right)$ assigns to the set $\left\{s_{-i}\right\} \times T_{-i}$. For every strategy $s_{i} \in S_{i}$, we denote by

$$
u_{i}\left(s_{i}, t_{i}\right):=\sum_{s_{-i} \in S_{-i}} \beta_{i}\left(t_{i}\right)\left(s_{-i}\right) u_{i}\left(s_{i}, s_{-i}\right)
$$

the expected utility for type $t_{i}$ of choosing strategy $s_{i}$. We say that strategy $s_{i}$ is rational for type $t_{i}$ if $u_{i}\left(s_{i}, t_{i}\right) \geq u_{i}\left(s_{i}^{\prime}, t_{i}\right)$ for every $s_{i}^{\prime} \in S_{i}$. 
Definition 3.1. (Belief in the opponents' rationality) Type $t_{i}$ is said to believe in the opponents' rationality if for every opponent $j$, and every strategy-type pair $\left(s_{j}, t_{j}\right) \in S_{j} \times T_{j}$ to which $t_{i}$ assigns positive probability, the strategy $s_{j}$ is rational for type $t_{j}$.

Definition 3.2. (Self-referential beliefs) Type $t_{i}$ is said to have self-referential beliefs if for every $p \in[0,1]$ and every event $E \subseteq S_{-i} \times T_{-i}$ which $t_{i}$ believes with probability $p$, type $t_{i}$ believes that all opponents believe that player $i$ believes $E$ with probability $p$.

Hence, a player with self-referential beliefs thinks that his opponents hold correct beliefs about his own beliefs. Now, let $j$ be an opponent of $i$, and let $E_{j} \subseteq S_{j} \times T_{j}$ be an event about player $j$. We say that $t_{i}$ believes $E_{j}$ with probability $p$ if $t_{i}$ assigns probability $p$ to the event

$$
E_{j} \times\left(\times_{k \neq i, j}\left(S_{k} \times T_{k}\right)\right) .
$$

Definition 3.3. (Projective beliefs) Type $t_{i}$ is said to have projective beliefs if for every pair of opponents $j, k$ and every event $E_{k} \subseteq S_{k} \times T_{k}$ about player $k$ : if $t_{i}$ believes $E_{k}$ with probability $p$, then $t_{i}$ believes that $j$ believes $E_{k}$ with probability $p$.

Intuitively, a player with projective beliefs projects his belief about an opponent on his other opponents. Of course, this condition only imposes restrictions if there are at least three players. Our last condition, conditionally independent beliefs, is taken from Brandenburger and Friedenberg (2006). It states that for every given profile $t_{-i}$ of opponents' types which is deemed possible by type $t_{i}$, his belief about the opponents' strategies conditional on $t_{-i}$ should be uncorrelated. In other words, any correlation in $t_{i}$ 's belief about the opponents' strategy choices should come from correlation in his belief about the opponents' types. The idea behind this condition is that types are assumed to choose their strategies independently since pre-play communication is not allowed. However, player $i$ 's belief about $j$ 's choice may still be dependent on his belief about $k$ 's choice if his belief about $j$ 's type is dependent on his belief about $k$ 's type. To formalize this condition, we need some terminology. Let $t_{-i}$ be a profile of opponents' types to which $t_{i}$ assigns positive probability, and let $s_{-i}$ be a profile of opponents' strategies. By

$$
\beta_{i}\left(t_{i}\right)\left(s_{-i} \mid t_{-i}\right):=\frac{\beta_{i}\left(t_{i}\right)\left(s_{-i}, t_{-i}\right)}{\beta_{i}\left(t_{i}\right)\left(t_{-i}\right)}
$$

we denote the probability that $t_{i}$ assigns to the strategy profile $s_{-i}$, conditional on the event that the opponents' types are given by $t_{-i}$. Here, $\beta_{i}\left(t_{i}\right)\left(t_{-i}\right)$ denotes the probability that $t_{i}$ assigns to the event that the opponents' types are $t_{-i}$. Similarly, for every opponent $j$, every type $t_{j}$ to which $t_{i}$ assigns positive probability, and every strategy $s_{j} \in S_{j}$, we denote by

$$
\beta_{i}\left(t_{i}\right)\left(s_{j} \mid t_{j}\right):=\frac{\beta_{i}\left(t_{i}\right)\left(s_{j}, t_{j}\right)}{\beta_{i}\left(t_{i}\right)\left(t_{j}\right)}
$$


the probability that $t_{i}$ assigns to strategy choice $s_{j}$, conditional on the event that $j$ 's type is $t_{j}$. Here, $\beta_{i}\left(t_{i}\right)\left(s_{j}, t_{j}\right)$ denotes the probability that $t_{i}$ assigns to the event that $j$ chooses $s_{j}$ and has type $t_{j}$, whereas $\beta_{i}\left(t_{i}\right)\left(t_{j}\right)$ is the probability that $t_{i}$ assigns to the event that $j$ has type $t_{j}$.

Definition 3.4. (Conditionally independent beliefs) Type $t_{i}$ is said to have conditionally independent beliefs if for every $t_{-i} \in T_{-i}$ with $\beta_{i}\left(t_{i}\right)\left(t_{-i}\right)>0$, and every $s_{-i} \in S_{-i}$ :

$$
\beta_{i}\left(t_{i}\right)\left(s_{-i} \mid t_{-i}\right)=\prod_{j \neq i} \beta_{i}\left(t_{i}\right)\left(s_{j} \mid t_{j}\right) .
$$

In fact, this condition combines the restrictions of conditional independence and sufficiency in Brandenburger and Friedenberg (2006). In their model, sufficiency states that player $i$ 's belief about player $j$ 's strategy choice should be independent of his belief about some other opponent's type. Obviously, our notion of conditionally independent beliefs satisfies this additional requirement. It should be clear that conditionally independent beliefs only imposes restrictions if there are at least three players.

As an abbreviation, we denote by BOR, SRB, PB and CIB the events that types believe in the opponents' rationality, have self-referential beliefs, have projective beliefs, and have conditionally independent beliefs, respectively. We say that type $t_{i}$ believes that every opponent satisfies BOR if $\beta_{i}\left(t_{i}\right)$ only assigns positive probability to opponents' types that satisfy BOR. Similarly, we define the events that $t_{i}$ believes that every opponent satisfies SRB, PB and CIB.

\section{Relation with Nash Equilibrium}

In this section we show that every type which satisfies BOR, SRB, and CIB, believes that every opponent satisfies BOR, SRB, PB and CIB, and chooses rationally, must choose a Nash equilibrium strategy. A profile $\left(\mu_{i}\right)_{i \in I}$ of probability distributions $\mu_{i} \in \Delta\left(S_{i}\right)$ is called a Nash equilibrium for the game $\Gamma$ if, for every player $i, \mu_{i}\left(s_{i}\right)>0$ only if $u_{i}\left(s_{i}, \mu_{-i}\right) \geq u_{i}\left(s_{i}^{\prime}, \mu_{-i}\right)$ for every $s_{i}^{\prime} \in S_{i}$. Here,

$$
u_{i}\left(s_{i}, \mu_{-i}\right):=\sum_{s_{-i} \in S_{-i}} \prod_{j \neq i} \mu_{j}\left(s_{j}\right) u_{i}\left(s_{i}, s_{-i}\right)
$$

denotes the expected utility for player $i$ of choosing $s_{i}$ if his belief about the opponents' strategies is given by $\mu_{-i}$. A strategy $s_{i}$ is called a Nash equilibrium strategy for $\Gamma$ if there is a Nash equilibrium $\left(\mu_{i}\right)_{i \in I}$ for $\Gamma$ such that $u_{i}\left(s_{i}, \mu_{-i}\right) \geq u_{i}\left(s_{i}^{\prime}, \mu_{-i}\right)$ for every $s_{i}^{\prime} \in S_{i}$. Note that every strategy that is assigned positive probability in some Nash equilibrium is also a Nash equilibrium strategy, but the converse is not true. Consider, for instance, the game in Figure 3. In this game, $\left(b, \frac{1}{2} c+\frac{1}{2} d\right)$ is a Nash equilibrium. Since $a$ is optimal against $\frac{1}{2} c+\frac{1}{2} d$, it follows that $a$ is a Nash equilibrium strategy. However, there is no Nash equilibrium $\left(\mu_{1}, \mu_{2}\right)$ in which $\mu_{1}$ assigns positive probability to $a$.

Before we prove our theorem on the relation with Nash equilibrium strategies, we first derive some implications of SRB, PB, CIB, and belief in these events. In each of these lemmas, we assume that $\mathcal{M}=\left(T_{i}, \beta_{i}\right)_{i \in I}$ is a finite epistemic model for a finite game $\Gamma$. 


\begin{tabular}{|l|l|l|}
\hline & $c$ & $d$ \\
\hline$a$ & 2,0 & 0,2 \\
\hline$b$ & 1,1 & 1,1 \\
\hline
\end{tabular}

Figure 3: Not every Nash equilibrium strategy is assigned positive probability in a Nash equilibrium

Lemma 4.1. Let $t_{i} \in T_{i}$ be a type with $S R B$. Then, $t_{i}$ believes that every opponent believes that $i$ 's type is $t_{i}$.

Proof. Choose an arbitrary event $E \subseteq S_{-i} \times T_{-i}$ for player $i$, and assume that $t_{i}$ believes $E$ with probability $p$. By SRB, $t_{i}$ believes that every opponent believes that $i$ believes $E$ with probability $p$. Since this holds for every $E$, and since the function $\beta_{i}: T_{i} \rightarrow \Delta\left(S_{-i} \times T_{-i}\right)$ in the epistemic model is one-to-one, $t_{i}$ believes that every opponent believes that $i$ 's type is $t_{i}$.

Lemma 4.2. Let $t_{i} \in T_{i}$ be a type with $S R B$ which believes that every opponent has $P B$. Then, $t_{i}$ has $P B$.

Proof. Suppose that $j$ and $k$ are two different opponents of player $i$. Let $E_{k} \subseteq S_{k} \times T_{k}$ be an event which $t_{i}$ believes with probability $p$, and let $t_{j} \in T_{j}$ be a type to which $t_{i}$ assigns positive probability. We show that $t_{j}$ believes $E_{k}$ with probability $p$ as well. Since $t_{i}$ has SRB, we know from Lemma 4.1 that $t_{j}$ believes that $i$ 's type is $t_{i}$. Since $t_{i}$ believes that $j$ has $\mathrm{PB}$, it must be the case that $t_{j}$ has $\mathrm{PB}$, and hence $t_{j}$ 's belief about player $k$ must be the same as $t_{i}$ 's belief about player $k$. Consequently, $t_{j}$ must believe $E_{k}$ with probability $p$. We may thus conclude that $t_{i}$ has PB.

Lemma 4.3. Let $t_{i}$ be a type with $S R B$ which believes that every opponent has $P B$ and $S R B$. Then, for every opponent $j$ there is some type $t_{j} \in T_{j}$ such that (1) $t_{i}$ believes for every $j \neq i$ that $j$ 's type is $t_{j}$, (2) for every $j \neq i$, type $t_{j}$ believes that $i$ 's type is $t_{i}$, and (3) for every $j, k \neq i$, type $t_{j}$ believes that $k$ 's type is $t_{k}$.

Proof. Suppose that $j \neq i$, and that $t_{i}$ assigns positive probability to type $t_{j} \in T_{j}$. By Lemma 4.1 we know that $t_{i}$ believes that $j$ believes that $i$ 's type is $t_{i}$. Hence, $t_{j}$ must believe that $i$ 's type is $t_{i}$. Since $t_{i}$ believes that $j$ has SRB, type $t_{j}$ must have SRB. By applying Lemma 4.1 to $t_{j}$, we may conclude that $t_{j}$ believes that $i$ believes that $j$ 's type is $t_{j}$. Since we have seen that $t_{j}$ believes that $i$ 's type is $t_{i}$, it follows that $t_{i}$ must believe that $j$ 's type is $t_{j}$. Hence, we have shown properties (1) and (2). It remains to show (3). By Lemma 4.2 we know that $t_{i}$ has PB. Suppose that $j, k \neq i$. Since $t_{i}$ believes that $k$ 's type is $t_{k}$, and since $t_{i}$ has $\mathrm{PB}$, it follows that $t_{i}$ 
believes that $j$ believes that $k$ 's type is $t_{k}$. Since $t_{i}$ believes that $j$ 's type is $t_{j}$, type $t_{j}$ believes that $k$ 's type is $t_{k}$. This completes the proof.

Lemma 4.4. Let $t_{i} \in T_{i}$ be a type that has $S R B$ and $C I B$, and believes that every opponent has $S R B, P B$ and CIB. Then, there is a probability distribution $\mu_{i} \in \Delta\left(S_{i}\right)$, and for every opponent $j$ a probability distribution $\mu_{j} \in \Delta\left(S_{j}\right)$ such that (1) $t_{i}$ believes that every opponents' strategy profile $\left(s_{j}\right)_{j \neq i}$ is chosen with probability $\prod_{j \neq i} \mu_{j}\left(s_{j}\right)$, (2) for every $j \neq i$, type $t_{i}$ believes that $j$ believes that every opponents' strategy profile $\left(s_{k}\right)_{k \neq j}$ is chosen with probability $\prod_{k \neq j} \mu_{k}\left(s_{k}\right)$, and (3) for every $j \neq i$, type $t_{i}$ believes that $j$ believes that $i$ believes that every opponents' strategy profile $\left(s_{j}\right)_{j \neq i}$ is chosen with probability $\prod_{j \neq i} \mu_{j}\left(s_{j}\right)$.

Proof. By Lemma 4.3, there is for every opponent $j$ some type $t_{j} \in T_{j}$ such that (1) $t_{i}$ believes for every $j \neq i$ that $j$ 's type is $t_{j}$, (2) for every $j \neq i$, type $t_{j}$ believes that $i$ 's type is $t_{i}$, and (3) for every $j, k \neq i$, type $t_{j}$ believes that $k$ 's type is $t_{k}$. Let $t_{-i}=\left(t_{j}\right)_{j \neq i}$. Since $t_{i}$ has CIB, it holds that

$$
\beta_{i}\left(t_{i}\right)\left(s_{-i} \mid t_{-i}\right)=\prod_{j \neq i} \beta_{i}\left(t_{i}\right)\left(s_{j} \mid t_{j}\right)
$$

for every $s_{-i} \in S_{-i}$. Now, define $\mu_{j}\left(s_{j}\right):=\beta_{i}\left(t_{i}\right)\left(s_{j} \mid t_{j}\right)$ for every $j \neq i$ and every $s_{j} \in S_{j}$. Then, by (4.1) and the fact that $t_{i}$ believes that every opponent $j$ has type $t_{j}$, type $t_{i}$ believes that every opponents' strategy profile $s_{-i}$ is chosen with probability $\prod_{j \neq i} \mu_{j}\left(s_{j}\right)$. We have thus shown property (1) of this lemma.

Now, choose some fixed opponent $j \neq i$. Since $t_{i}$ believes that $j$ has CIB, and $t_{i}$ believes that $j$ 's type is $t_{j}$, type $t_{j}$ has CIB. Hence,

$$
\beta_{j}\left(t_{j}\right)\left(s_{-j} \mid t_{-j}\right)=\prod_{k \neq j} \beta_{j}\left(t_{j}\right)\left(s_{k} \mid t_{k}\right)
$$

for every opponents' strategy profile $s_{-j}$. Choose some arbitrary player $k \notin\{i, j\}$. Since, by Lemma $4.2, t_{i}$ has PB and believes that $j$ 's type is $t_{j}$, type $t_{i}$ 's belief about $k$ 's strategy choice must be equal to $t_{j}$ 's belief about $k$ 's strategy choice, and hence $\beta_{j}\left(t_{j}\right)\left(s_{k} \mid t_{k}\right)=\beta_{i}\left(t_{i}\right)\left(s_{k} \mid t_{k}\right)=$ $\mu_{k}\left(s_{k}\right)$ for every $s_{k} \in S_{k}$. Define $\mu_{i}\left(s_{i}\right):=\beta_{j}\left(t_{j}\right)\left(s_{i} \mid t_{i}\right)$ for every $s_{i} \in S_{i}$. Together with (4.2), we may then conclude that $t_{j}$ believes that every opponents' strategy profile $s_{-j}$ is chosen with probability $\prod_{k \neq j} \mu_{k}\left(s_{k}\right)$. So, $t_{i}$ believes that $j$ believes that every opponents' strategy profile $s_{-j}$ is chosen with probability $\prod_{k \neq j} \mu_{k}\left(s_{k}\right)$.

Finally, choose some arbitrary player $k \notin\{i, j\}$. Since $t_{i}$ believes that $k$ has CIB, and believes that $k$ 's type is $t_{k}$, type $t_{k}$ must have CIB. Hence,

$$
\beta_{k}\left(t_{k}\right)\left(s_{-k} \mid t_{-k}\right)=\prod_{l \neq k} \beta_{k}\left(t_{k}\right)\left(s_{l} \mid t_{l}\right)
$$

for every $s_{-k} \in S_{-k}$. Let $l \notin\{i, k\}$. By Lemma 4.2 we know that $t_{i}$ has PB. Since $t_{i}$ has PB, and believes that $k$ 's type is $t_{k}$, type $t_{i}$ 's belief about l's strategy choice must be equal to $k$ 's belief about l's strategy choice, and hence $\beta_{k}\left(t_{k}\right)\left(s_{l} \mid t_{l}\right)=\beta_{i}\left(t_{i}\right)\left(s_{l} \mid t_{l}\right)=\mu_{l}\left(s_{l}\right)$ for every $s_{l} \in S_{l}$. 
Since $t_{i}$ believes that $j$ has $\mathrm{PB}$, and believes that $j$ has type $t_{j}$, type $t_{j}$ must have PB. Hence, $t_{j}$ 's belief about $i$ 's strategy choice must be equal to $k$ 's belief about $i$ 's strategy choice, which implies that $\beta_{k}\left(t_{k}\right)\left(s_{i} \mid t_{i}\right)=\beta_{j}\left(t_{j}\right)\left(s_{i} \mid t_{i}\right)=\mu_{i}\left(s_{i}\right)$. Combined with (4.3) we obtain that $t_{k}$ believes that every opponents' strategy profile $s_{-k}$ is chosen with probability $\prod_{l \neq k} \mu_{l}\left(s_{l}\right)$. So, $t_{i}$ believes that every $k \neq j$ believes that every opponents' strategy profile $s_{-k}$ is chosen with probability $\prod_{l \neq k} \mu_{l}\left(s_{l}\right)$. Hence, we have shown property (2).

Since we already know from Lemma 4.1 that $t_{i}$ believes that every opponent $j$ believes that $i$ 's type is $t_{i}$, property (3) follows immediately. This completes the proof.

We are now ready to prove our main theorem.

Theorem 4.5. Let $\mathcal{M}=\left(T_{i}, \beta_{i}\right)_{i \in I}$ be a finite epistemic model for a finite game $\Gamma$. Let $t_{i} \in T_{i}$ be a type that satisfies BOR, SRB and CIB, and believes that every opponent satisfies BOR, $S R B, P B$ and $C I B$. Then, every strategy that is rational for $t_{i}$ is a Nash equilibrium strategy for $\Gamma$.

Proof. Let $t_{i}$ be a type that satisfies BOR, SRB and CIB, and believes that every opponent satisfies BOR, SRB, PB and CIB. By Lemma 4.4, there is a probability distribution $\mu_{i} \in \Delta\left(S_{i}\right)$, and for every opponent $j$ a probability distribution $\mu_{j} \in \Delta\left(S_{j}\right)$ such that (1) $t_{i}$ believes that every opponents' strategy profile $\left(s_{j}\right)_{j \neq i}$ is chosen with probability $\prod_{j \neq i} \mu_{j}\left(s_{j}\right)$, (2) for every $j \neq i$, type $t_{i}$ believes that $j$ believes that every opponents' strategy profile $\left(s_{k}\right)_{k \neq j}$ is chosen with probability $\prod_{k \neq j} \mu_{k}\left(s_{k}\right)$, and (3) for every $j \neq i$, type $t_{i}$ believes that $j$ believes that $i$ believes that every opponents' strategy profile $\left(s_{j}\right)_{j \neq i}$ is chosen with probability $\prod_{j \neq i} \mu_{j}\left(s_{j}\right)$. We show that $\left(\mu_{j}\right)_{j \in I}$ is a Nash equilibrium.

Suppose first that $j \neq i$ and that $\mu_{j}\left(s_{j}\right)>0$ for some $s_{j} \in S_{j}$. Since $t_{i}$ believes in $j$ 's rationality, and, by (2), believes that $j$ 's belief about the opponents' strategy choices is given by $\mu_{-j}=\left(\mu_{k}\right)_{k \neq j}$, it follows that $s_{j}$ must be optimal against $\mu_{-j}$. Finally, let $\mu_{i}\left(s_{i}\right)>0$ for some $s_{i} \in S_{i}$. Choose some arbitrary opponent $j$. Since, by (2), $t_{i}$ 's belief about $j$ 's belief about the opponents' strategy choice is given by $\mu_{-j}$, type $t_{i}$ believes that $j$ believes that $i$ chooses $s_{i}$ with positive probability. Since $t_{i}$ believes that $j$ believes in $i$ 's rationality, and since, by (3), $t_{i}$ 's belief about $j$ 's belief about $i$ 's belief about the opponents' strategy choices is given by $\mu_{-i}$, it follows that $s_{i}$ must be optimal against $\mu_{-i}$. Hence, $\left(\mu_{j}\right)_{j \in I}$ is a Nash equilibrium.

Now, choose some strategy $s_{i}$ that is rational for $t_{i}$. Since, by (1), $t_{i}$ 's belief about the opponents' strategy choices is $\mu_{-i}$, strategy $s_{i}$ must be optimal against $\mu_{-i}$. As $\left(\mu_{i}, \mu_{-i}\right)$ is a Nash equilibrium, $s_{i}$ is a Nash equilibrium strategy. This completes the proof.

Our last result shows that every Nash equilibrium strategy can be chosen rationally by a type that satisfies BOR, SRB and CIB, and believes that his opponents satisfy BOR, SRB, PB and CIB. In particular, the combination of these seven events is shown to be possible. 
Theorem 4.6. Let $\Gamma$ be a finite game, and let $s_{i}$ be a Nash equilibrium strategy for player $i$ in $\Gamma$. Then, there exists a finite epistemic model $\mathcal{M}=\left(T_{i}, \beta_{i}\right)_{i \in I}$ for $\Gamma$, and a type $t_{i} \in T_{i}$, such that $s_{i}$ is rational for $t_{i}$, type $t_{i}$ satisfies BOR, SRB, and CIB, and believes that every opponent satisfies BOR, SRB, PB and CIB.

Proof. Let $s_{i}$ be a Nash equilibrium strategy for $\Gamma$. Then, there is a Nash equilibrium $\left(\mu_{j}\right)_{j \in I}$ for $\Gamma$ such that $s_{i}$ is optimal for $i$ against $\mu_{-i}$. We define the epistemic model $\mathcal{M}=\left(T_{i}, \beta_{i}\right)_{i \in I}$ as follows: Define $T_{j}:=\left\{\hat{t}_{j}\right\}$ for every player $j$, and let $\beta_{j}\left(\hat{t}_{j}\right)$ be the probability distribution on $S_{-j} \times T_{-j}$ given by

$$
\beta_{j}\left(\hat{t}_{j}\right)\left(s_{-j}, t_{-j}\right):=\left\{\begin{array}{r}
\prod_{k \neq j} \mu_{k}\left(s_{k}\right), \text { if } t_{-j}=\hat{t}_{-j} \\
0, \text { otherwise. }
\end{array}\right.
$$

We show that for every player $j$, the type $\hat{t}_{j}$ satisfies BOR, SRB, PB and CIB. Since $\hat{t}_{i}$ believes, for every opponent $j$, that $j$ 's type is $\hat{t}_{j}$, it would follow that $\hat{t}_{i}$ believes that every opponent satisfies these four conditions as well.

BOR: Let $k$ be an opponent for $j$, and let $s_{k}$ be a strategy for $k$ with $\beta_{j}\left(\hat{t}_{j}\right)\left(s_{k}, \hat{t}_{k}\right)>0$. Then, $\mu_{k}\left(s_{k}\right)>0$. Since $\left(\mu_{j}\right)_{j \in I}$ is a Nash equilibrium, $s_{k}$ is optimal for $k$ against $\mu_{-k}$, and hence $s_{k}$ is rational for $\hat{t}_{k}$. Therefore, $\hat{t}_{j}$ satisfies BOR.

SRB: By construction, $\hat{t}_{j}$ believes that every opponent $k$ has type $\hat{t}_{k}$. Moreover, every such type $\hat{t}_{k}$ believes that $j$ 's type is $\hat{t}_{j}$. Hence, $\hat{t}_{j}$ believes that every opponent believes that $j$ 's type is $\hat{t}_{j}$. Now, suppose that $\hat{t}_{j}$ believes an event $E$ with probability $p$. Then, since $\hat{t}_{j}$ believes that every opponent believes that $j$ 's type is $\hat{t}_{j}$, type $\hat{t}_{j}$ believes that every opponent believes that $j$ believes $E$ with probability $p$. Consequently, $\hat{t}_{j}$ satisfies SRB.

PB: Let $k, l$ be two different opponents for $j$, let $E_{k} \subseteq S_{k} \times T_{k}$, and suppose that $\hat{t}_{j}$ believes $E_{k}$ with probability $p$. By construction, $\hat{t}_{j}$ believes that $l$ 's type is $\hat{t}_{l}$, and $\hat{t}_{l}$ 's belief about player $k$ is the same as $\hat{t}_{j}$ 's belief about player $k$. Hence, $\hat{t}_{j}$ believes that $l$ believes $E_{k}$ with probability $p$. We may thus conclude that $\hat{t}_{j}$ satisfies PB.

CIB: It follows immediately from the construction of the epistemic model that $\hat{t}_{j}$ satisfies CIB.

Hence, we may conclude that every $\hat{t}_{j}$ satisfies BOR, SRB, PB and CIB. This implies that $\hat{t}_{i}$ satisfies these four conditions, and believes that every opponent satisfies these four conditions too. Recall that the Nash equilibrium $\left(\mu_{j}\right)_{j \in I}$ was chosen such that $s_{i}$ is optimal for $i$ against $\mu_{-i}$. Since $\mu_{-i}$ is $\hat{t}_{i}$ 's belief about the opponents' strategy choices, $s_{i}$ is rational for $\hat{t}_{i}$. This completes the proof.

\section{No Conditions Can Be Dropped}

In Theorem 4.5 we have shown that the conditions BOR, SRB, CIB, and belief in BOR, SRB, $\mathrm{PB}$ and $\mathrm{CIB}$, lead to Nash equilibrium strategy choices. So, in total we have seven conditions 


\begin{tabular}{|l|l|l|}
\hline & $d$ & $e$ \\
\hline$a$ & 2,0 & 1,1 \\
\hline$b$ & 1,0 & 2,1 \\
\hline$c$ & 0,1 & 0,0 \\
\hline
\end{tabular}

Figure 4: BOR and belief in BOR cannot be dropped

\begin{tabular}{|l|l|l|l|}
\hline & $d$ & $e$ & $f$ \\
\hline$a$ & 3,0 & 0,3 & 0,0 \\
\hline$b$ & 0,3 & 3,0 & 0,0 \\
\hline$c$ & 2,0 & 2,0 & 2,2 \\
\hline
\end{tabular}

Figure 5: SRB and belief in SRB cannot be dropped

that we impose on a player's beliefs. In this section we prove that this result no longer holds if we drop one of these seven conditions. In particular, we show that these seven conditions are logically independent.

Dropping BOR: Consider the two-player game in Figure 4, where player 1 chooses the rows and player 2 the columns. Construct an epistemic model $\mathcal{M}$ such that $T_{1}=\left\{t_{1}\right\}, T_{2}=\left\{t_{2}\right\}$, $\beta_{1}\left(t_{1}\right)$ assigns probability 1 to $\left(d, t_{2}\right)$, and $\beta_{2}\left(t_{2}\right)$ assigns probability 1 to $\left(a, t_{1}\right)$. Then, $t_{1}$ does not BOR, since $d$ is not optimal for $t_{2}$. On the other hand, $t_{1}$ believes that player 2 BOR, since $a$ is optimal for $t_{1}$. Type $t_{1}$ has SRB since $t_{1}$ believes that player 2 believes that his type is $t_{1}$. Similarly, $t_{2}$ has SRB, and hence $t_{1}$ believes in SRB. Clearly, $t_{1}$ has CIB, and believes that player 2 has PB and CIB, since these conditions are automatically satisfied for two players. Hence, $t_{1}$ does not BOR, but satisfies the other six conditions. Strategy $a$ is rational for $t_{1}$, but $a$ is not a Nash equilibrium strategy.

Dropping belief in BOR: Consider again the two-player game in Figure 4. Construct an epistemic model $\mathcal{M}$ such that $T_{1}=\left\{t_{1}\right\}, T_{2}=\left\{t_{2}\right\}, \beta_{1}\left(t_{1}\right)$ assigns probability 1 to $\left(d, t_{2}\right)$, and $\beta_{2}\left(t_{2}\right)$ assigns probability 1 to $\left(c, t_{1}\right)$. Then, $t_{1}$ does not believe in BOR, since $c$ is not optimal for $t_{1}$. However, $t_{1}$ satisfies the other six conditions. Strategy $a$ is optimal for $t_{1}$, but $a$ is not a Nash equilibrium strategy.

Dropping SRB: Consider the two-player game in Figure 5. It can be shown that $(c, f)$ is the only Nash equilibrium, and hence $c$ is the only Nash equilibrium strategy for player 1 . Construct an epistemic model $\mathcal{M}$ such that $T_{1}=\left\{t_{1}^{a}, t_{1}^{b}\right\}, T_{2}=\left\{t_{2}\right\}, \beta_{1}\left(t_{1}^{a}\right)$ assigns probability 1 to $\left(d, t_{2}\right), \beta_{1}\left(t_{1}^{b}\right)$ assigns probability 1 to $\left(e, t_{2}\right)$, and $\beta_{2}\left(t_{2}\right)$ assigns probability $\frac{1}{2}$ to $\left(a, t_{1}^{a}\right)$ and probability $\frac{1}{2}$ to $\left(b, t_{1}^{b}\right)$. Then, $t_{1}^{a}$ does not have SRB, since $t_{1}^{a}$ believes that player 2 believes with probability $\frac{1}{2}$ that his type is $t_{1}^{b}$ and not $t_{1}^{a}$. However, $t_{1}^{a}$ satisfies the other six conditions. Strategy $a$ is optimal for $t_{1}^{a}$, but $a$ is not a Nash equilibrium strategy. 


\begin{tabular}{|l|l|l|l|}
\hline$g$ & $d$ & $e$ & $f$ \\
\hline$a$ & $3,3,0$ & $3,0,3$ & $0,2,0$ \\
\hline$b$ & $0,0,0$ & $0,0,0$ & $0,2,0$ \\
\hline$c$ & $2,0,0$ & $2,0,0$ & $2,2,0$ \\
\hline
\end{tabular}

\begin{tabular}{|l|l|l|l|}
\hline$h$ & $d$ & $e$ & $f$ \\
\hline$a$ & $0,0,0$ & $0,3,3$ & $0,2,0$ \\
\hline$b$ & $0,0,0$ & $3,3,0$ & $0,2,0$ \\
\hline$c$ & $2,0,0$ & $2,0,0$ & $2,2,0$ \\
\hline
\end{tabular}

\begin{tabular}{|l|l|l|l|}
\hline$i$ & $d$ & $e$ & $f$ \\
\hline$a$ & $0,0,2$ & $0,0,2$ & $0,2,2$ \\
\hline$b$ & $0,0,2$ & $0,0,2$ & $0,2,2$ \\
\hline$c$ & $2,0,2$ & $2,0,2$ & $2,2,2$ \\
\hline
\end{tabular}

Figure 6: Belief in PB cannot be dropped

\begin{tabular}{|l|l|l|l|}
\hline$g$ & $d$ & $e$ & $f$ \\
\hline$a$ & $0,0,3$ & $0,0,3$ & $3,3,3$ \\
\hline$b$ & $0,0,0$ & $0,0,0$ & $3,3,3$ \\
\hline$c$ & $3,3,3$ & $3,3,3$ & $3,3,3$ \\
\hline
\end{tabular}

\begin{tabular}{|l|l|l|l|}
\hline$h$ & $d$ & $e$ & $f$ \\
\hline$a$ & $0,0,2$ & $0,0,0$ & $0,0,0$ \\
\hline$b$ & $0,0,0$ & $0,0,2$ & $0,0,0$ \\
\hline$c$ & $0,0,0$ & $0,0,0$ & $0,0,0$ \\
\hline
\end{tabular}

\begin{tabular}{|l|l|l|l|}
\hline$i$ & $d$ & $e$ & $f$ \\
\hline$a$ & $0,0,0$ & $0,0,0$ & $3,3,3$ \\
\hline$b$ & $0,0,3$ & $0,0,3$ & $3,3,3$ \\
\hline$c$ & $3,3,3$ & $3,3,3$ & $3,3,3$ \\
\hline
\end{tabular}

Figure 7: CIB and belief in CIB cannot be dropped

Dropping belief in SRB: Consider again the two-player game in Figure 5. Recall that $(c, f)$ is the only Nash equilibrium, and hence $f$ is the only Nash equilibrium strategy for player 2. Construct an epistemic model $\mathcal{M}$ such that $T_{1}=\left\{t_{1}^{a}, t_{1}^{b}\right\}, T_{2}=\left\{t_{2}\right\}, \beta_{1}\left(t_{1}^{a}\right)$ assigns probability 1 to $\left(d, t_{2}\right), \beta_{1}\left(t_{1}^{b}\right)$ assigns probability 1 to $\left(e, t_{2}\right)$, and $\beta_{2}\left(t_{2}\right)$ assigns probability $\frac{1}{2}$ to $\left(a, t_{1}^{a}\right)$ and probability $\frac{1}{2}$ to $\left(b, t_{1}^{b}\right)$. Then, $t_{2}$ does not believe in SRB, since $t_{1}^{a}$ and $t_{1}^{b}$ do not have SRB. However, $t_{2}$ satisfies the other six conditions. Strategy $d$ is optimal for $t_{2}$, but $d$ is not a Nash equilibrium strategy.

Dropping belief in PB: Consider the three-player game in Figure 6. Here, player 1 chooses the row, player 2 chooses the column, and player 3 chooses the matrix $(g, h$ or $i)$. Construct an epistemic model $\mathcal{M}$ such that $T_{1}=\left\{t_{1}\right\}, T_{2}=\left\{t_{2}\right\}, T_{3}=\left\{t_{3}\right\}, \beta_{1}\left(t_{1}\right)$ assigns probability 1 to $\left(\left(e, t_{2}\right),\left(g, t_{3}\right)\right), \beta_{2}\left(t_{2}\right)$ assigns probability 1 to $\left(\left(a, t_{1}\right),\left(h, t_{3}\right)\right)$, and $\beta_{3}\left(t_{3}\right)$ assigns probability 1 to $\left(\left(a, t_{1}\right),\left(e, t_{2}\right)\right)$. Type $t_{3}$ does not believe in $\mathrm{PB}$, since $t_{1}$ does not have PB. In order to see this, note that $t_{1}$ believes that player 3 chooses $g$, whereas $t_{1}$ believes that player 2 believes that player 3 chooses $h$. It can easily be verified that $t_{3}$ satisfies the other six conditions.

Strategy $g$ is rational for type $t_{3}$. However, we will show that $g$ is not a Nash equilibrium strategy. Suppose, contrary to what we want to show, that $g$ would be a Nash equilibrium strategy. Then, there would be a Nash equilibrium $\left(\mu_{1}, \mu_{2}, \mu_{3}\right)$ such that $g$ would be optimal against $\left(\mu_{1}, \mu_{2}\right)$. Strategy $g$ can only be optimal against $\left(\mu_{1}, \mu_{2}\right)$ if $\mu_{1}(a)>0$ and $\mu_{2}(e)>0$. Since $\left(\mu_{1}, \mu_{2}, \mu_{3}\right)$ is a Nash equilibrium, this implies that $a$ is optimal against $\left(\mu_{2}, \mu_{3}\right)$ and $e$ is optimal against $\left(\mu_{1}, \mu_{3}\right)$. This, in turn, implies that $\mu_{3}(g) \geq \frac{2}{3}$ and $\mu_{3}(h) \geq \frac{2}{3}$, which is clearly impossible. Hence, $g$ is not a Nash equilibrium strategy.

Dropping CIB: Consider the three-player game in Figure 7. Construct an epistemic model $\mathcal{M}$ such that $T_{1}=\left\{t_{1}\right\}, T_{2}=\left\{t_{2}\right\}, T_{3}=\left\{t_{3}\right\}, \beta_{1}\left(t_{1}\right)$ assigns probability $\frac{1}{2}$ to $\left(\left(d, t_{2}\right),\left(h, t_{3}\right)\right)$ and probability $\frac{1}{2}$ to $\left(\left(e, t_{2}\right),\left(h, t_{3}\right)\right), \beta_{2}\left(t_{2}\right)$ assigns probability $\frac{1}{2}$ to $\left(\left(a, t_{1}\right),\left(h, t_{3}\right)\right)$ and probability 
$\frac{1}{2}$ to $\left(\left(b, t_{1}\right),\left(h, t_{3}\right)\right)$, and $\beta_{3}\left(t_{3}\right)$ assigns probability $\frac{1}{2}$ to $\left(\left(a, t_{1}\right),\left(d, t_{2}\right)\right)$ and probability $\frac{1}{2}$ to $\left(\left(b, t_{1}\right),\left(e, t_{2}\right)\right)$. Then, $t_{3}$ does not have CIB. It may be verified that $t_{3}$ satisfies the other six conditions.

Strategy $h$ is rational for $t_{3}$. However, we will show that $h$ is not a Nash equilibrium strategy. Assume, namely, that $\left(\mu_{1}, \mu_{2}, \mu_{3}\right)$ would be a Nash equilibrium such that $h$ would be optimal against $\left(\mu_{1}, \mu_{2}\right)$. If $\mu_{1}(a) \leq \frac{1}{2}$, it can be shown that $u_{3}\left(h, \mu_{1}, \mu_{2}\right)<u_{3}\left(i, \mu_{1}, \mu_{2}\right)$. If $\mu_{1}(a) \geq \frac{1}{2}$, it can be shown that $u_{3}\left(h, \mu_{1}, \mu_{2}\right)<u_{3}\left(g, \mu_{1}, \mu_{2}\right)$. Hence, $h$ can never be optimal against $\left(\mu_{1}, \mu_{2}\right)$, and therefore $h$ is not a Nash equilibrium strategy.

Dropping belief in CIB: Consider again the three-player game in Figure 7. Construct an epistemic model $\mathcal{M}$ such that $T_{1}=\left\{t_{1}\right\}, T_{2}=\left\{t_{2}\right\}, T_{3}=\left\{t_{3}\right\}, \beta_{1}\left(t_{1}\right)$ assigns probability $\frac{1}{2}$ to $\left(\left(d, t_{2}\right),\left(h, t_{3}\right)\right)$ and probability $\frac{1}{2}$ to $\left(\left(e, t_{2}\right),\left(h, t_{3}\right)\right), \beta_{2}\left(t_{2}\right)$ assigns probability $\frac{1}{2}$ to $\left(\left(a, t_{1}\right),\left(h, t_{3}\right)\right)$ and probability $\frac{1}{2}$ to $\left(\left(b, t_{1}\right),\left(h, t_{3}\right)\right)$, and $\beta_{3}\left(t_{3}\right)$ assigns probability $\frac{1}{2}$ to $\left(\left(a, t_{1}\right),\left(d, t_{2}\right)\right)$ and probability $\frac{1}{2}$ to $\left(\left(b, t_{1}\right),\left(e, t_{2}\right)\right)$. Then, $t_{1}$ does not believe in CIB, since $t_{3}$ does not have CIB. It may be verified that $t_{1}$ satisfies the other six conditions.

Strategy $a$ is rational for $t_{1}$. However, we will show that $a$ is not a Nash equilibrium strategy. We have seen above that $h$ is not a Nash equilibrium strategy. In particular, there is no Nash equilibrium that assigns positive probability to $h$. But then, $a$ cannot be optimal in a Nash equilibrium, and hence $a$ is not a Nash equilibrium strategy.

\section{Comparison with Other Models}

In this section we compare our sufficient conditions for Nash equilibrium with those proposed in Aumann and Brandenburger (1995), Asheim (2006) and Brandenburger and Dekel (1987). One important difference with the latter foundations is that our model only imposes restrictions on the beliefs of a single player, while the other models simultaneously impose restrictions on the beliefs of all players. In other words, we view the game from the perspective of a single player, whereas the other foundations do not. We shall now discuss the similarities and differences with the other models in some more detail.

\subsection{Aumann and Brandenburger's Model}

Aumann and Brandenburger (1995) (AB from now on) make a distinction between the case of two players and the case of more than two players, and provide sufficient conditions for Nash equilibrium for both cases. In AB's model, a type for player $i$ does not only specify $i$ 's belief hierarchy, but also $i$ 's strategy choice and $i$ 's utility function. It is therefore possible to say that type $t_{i}$ is rational. AB's theorem for two-player games may be formulated as follows: Consider a pair $\left(u_{1}, u_{2}\right)$ of utility functions, a pair $\left(\mu_{1}, \mu_{2}\right) \in \Delta\left(S_{1}\right) \times \Delta\left(S_{2}\right)$ of probability distributions over strategy choices, and a pair $\left(t_{1}, t_{2}\right)$ of types. If at $\left(t_{1}, t_{2}\right)$ it is true that $(1)$ both players are rational and believe that the opponent is rational, (2) both players $i$ have utility function $u_{i}$ and believe that opponent $j$ has utility function $u_{j}$, and (3) both players $i$ have belief $\mu_{j}$ about 
$j$ 's strategy choice, and believe that $j$ has belief $\mu_{i}$ about $i$ 's strategy choice, then $\left(\mu_{1}, \mu_{2}\right)$ is a Nash equilibrium with respect to $\left(u_{1}, u_{2}\right)$.

A key assumption in AB's theorem is that $t_{i}$ believes that player $j$ has belief $\mu_{i}$ about $i$ 's strategy choice. That is, $t_{i}$ cannot assign positive probability to types $t_{j}$ and $t_{j}^{\prime}$ that have different beliefs about $i$ 's strategy choice. In our model, this property follows from our assumption that $t_{i}$ has SRB and believes that $j$ has SRB (see Lemma 4.3), but, in contrast to AB, we do not impose this property explicitly.

For the case of more than two players, AB add the following two conditions: (4) at the type profile $\left(t_{i}\right)_{i \in I}$ the types' belief hierarchies are derived from a common prior probability distribution on the set of type profiles, and (5) at $\left(t_{i}\right)_{i \in I}$ there is common belief in the profile $\left(\beta_{i}\right)_{i \in I} \in \times_{i \in I} \Delta\left(S_{-i}\right)$ of beliefs about the opponents' strategies. Here, by common belief we mean that $t_{i}$ 's belief about the opponents' choices is $\beta_{i}$, that $t_{i}$ believes that every opponent $j$ has belief $\beta_{j}$ about the other players' choices, that $t_{i}$ believes that every opponent $j$ believes that every other player $k$ has belief $\beta_{k}$ about the opponents' choices, and so on. By adding these conditions (4) and (5), $\mathrm{AB}$ are able to show that there is some profile $\left(\mu_{i}\right)_{i \in I} \in \times_{i \in I} \Delta\left(S_{i}\right)$ of probability distributions over strategy choices such that for every player $i$, type $t_{i}$ 's belief about the opponents' choices is given by $\left(\mu_{j}\right)_{j \neq i}$, and that $\left(\mu_{i}\right)_{i \in I}$ is a Nash equilibrium with respect to $\left(u_{i}\right)_{i \in I}$. In particular, the conditions (4) and (5) imply that $i$ 's belief about $j$ 's choice is independent from $i$ 's belief about $k$ 's choice, and that two different players $i$ and $j$ have the same belief about $k$ 's choice. These two properties are crucial for their proof. In our model, these two properties follow from the assumption that $t_{i}$ has SRB and CIB, and believes that every opponent has SRB, PB and CIB (see Lemma 4.4). Hence, one could say that in our model the conditions of CIB and belief in PB and CIB play a similar role as the conditions (4) and (5) in $\mathrm{AB}$.

\subsection{Asheim's Model}

Asheim (2006), on page 5 of his book, provides a sufficient condition for Nash equilibrium for the case of two players. He basically uses the same epistemic model as we do, and his result may be stated as follows: Consider a pair $\left(t_{1}, t_{2}\right)$ of types, and assume that (1) $t_{1}$ and $t_{2}$ BOR, and (2) for both players $i$, type $t_{i}$ believes that his opponent has type $t_{j}$. Then, $t_{1}$ 's belief about 2 's choice and $t_{2}$ 's belief about 1's choice constitute a Nash equilibrium. The key assumption is that $t_{i}$ assigns probability one to a single type $t_{j}$ for his opponent, and that $t_{j}$ believes that $i$ 's type is $t_{i}$. In our setting, this property follows from the assumption that $t_{i}$ has SRB and believes that $j$ has SRB (see Lemma 4.3), but we do not impose this condition explicitly as Asheim does.

\subsection{Brandenburger and Dekel's Model}

Brandenburger and Dekel (1987) (BD from now on) use a model which substantially differs from ours and the ones above. BD assume that there is a finite state space $\Omega$, that every player $i$ holds 
a prior belief $P_{i} \in \Delta(\Omega)$, that for every player $i$ there is an information partition $\mathcal{H}_{i}$ of $\Omega$, and that for every $H_{i} \in \mathcal{H}_{i}$ there is a conditional belief $P_{i}\left(\cdot \mid H_{i}\right) \in \Delta\left(H_{i}\right)$ which is derived from $P_{i}$ by Bayes' rule whenever possible. A strategy map for player $i$ is an $\mathcal{H}_{i}$-measurable map $f_{i}$ from $\Omega$ to $S_{i}$. A profile $\left(f_{i}\right)_{i \in I}$ of strategy maps is called an a posteriori equilibrium if for every player $i$, at every $H_{i} \in \mathcal{H}_{i}$ the prescribed strategy is optimal given the conditional belief $P_{i}\left(\cdot \mid H_{i}\right)$ and the opponents' strategy maps $\left(f_{j}\right)_{j \neq i}$. Hence, it is implicitly assumed that players have correct beliefs about the opponents' strategy maps, and that there is common belief in this event. The prior beliefs $\left(P_{i}\right)_{i \in I}$ are called concordant if, for every choice of the strategy maps, two different players $i$ and $j$ have the same prior belief about $k$ 's choice. The information partitions $\left(\mathcal{H}_{j}\right)_{j \neq i}$

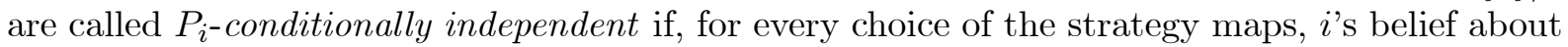
$j$ 'choice is independent from $i$ 's belief about $k$ 's choice. The information partitions $\left(\mathcal{H}_{j}\right)_{j \neq i}$ are called $P_{i}$-informationally independent if, for every choice of the strategy maps, $i$ 's belief about the opponents' choices does not depend on the information set $H_{i}$.

BD's theorem on page 1401 can now be stated as follows: Suppose that the prior beliefs $\left(P_{i}\right)_{i \in I}$ and the information partitions $\left(\mathcal{H}_{i}\right)_{i \in I}$ are such that $(1)$ the prior beliefs $\left(P_{i}\right)_{i \in I}$ are concordant, and for every $i$, the information partitions $\left(\mathcal{H}_{j}\right)_{j \neq i}$ are $(2) P_{i}$-conditionally independent and (3) $P_{i}$-informationally independent. Then, for every a posteriori equilibrium there is a profile $\left(\mu_{i}\right)_{i \in I} \in \times_{i \in I} \Delta\left(S_{i}\right)$ of probability distributions over strategy choices such that, for every $i$, player $i$ 's prior belief about the opponents' choices is given by $\left(\mu_{j}\right)_{j \neq i}$, and $\left(\mu_{i}\right)_{i \in I}$ is a Nash equilibrium.

In BD's theorem, concordance of the prior beliefs guarantees that $i$ and $j$ have the same beliefs about $k$ 's choice, and is therefore similar to our condition of PB. The assumption in $\mathrm{BD}$ that the information partitions $\left(\mathcal{H}_{j}\right)_{j \neq i}$ are $P_{i}$-informationally independent guarantees that $i$ 's belief about his opponents is independent of his information state, and therefore $i$ believes that his opponents are right about his own beliefs. (Recall the implicit assumption that players have correct beliefs about the opponents' strategy maps, and that there is common belief in this event). The assumption of $P_{i}$-informational independence has therefore the same effect as SRB is our model. In $\mathrm{BD}, P_{i}$-conditional independence guarantees that $i$ 's belief about $j$ 's choice is independent from $i$ 's belief about $k$ 's choice. In our model, this property is implied by the conditions of SRB, CIB, and belief in SRB, PB and CIB (see Lemma 4.4). However, we do not impose this property explicitly.

\section{References}

[1] Asheim, G.B. (2006), The consistent preferences approach to deductive reasoning in games, Theory and Decision Library, Springer, Dordrecht, The Netherlands.

[2] Aumann, R. and A. Brandenburger (1995), Epistemic conditions for Nash equilibrium, Econometrica 63, 1161-1180. 
[3] Bernheim, D. (1984), Rationalizable strategic behavior, Econometrica 52, 1007-1028.

[4] Brandenburger, A. and E. Dekel (1987), Rationalizability and correlated equilibria, Econometrica 55, 1391-1402.

[5] Brandenburger, A. and A. Friedenberg (2006), Intrinsic correlation in games, Available at http://pages.stern.nyu.edu/ abranden/

[6] Myerson, R. (1991), Game Theory: Analysis of Conflict, Harvard University Press, Cambridge, Massachusetts, London, England.

[7] Nash, J. (1951), Non-cooperative games, Annals of Mathematics 54, 286-295.

[8] Pearce, D. (1984), Rationalizable strategic behavior and the problem of perfection, Econometrica 52, 1029-1050.

[9] Tan, T. and S.R.C. Werlang (1988), The bayesian foundations of solution concepts of games, Journal of Economic Theory 45, 370-391. 